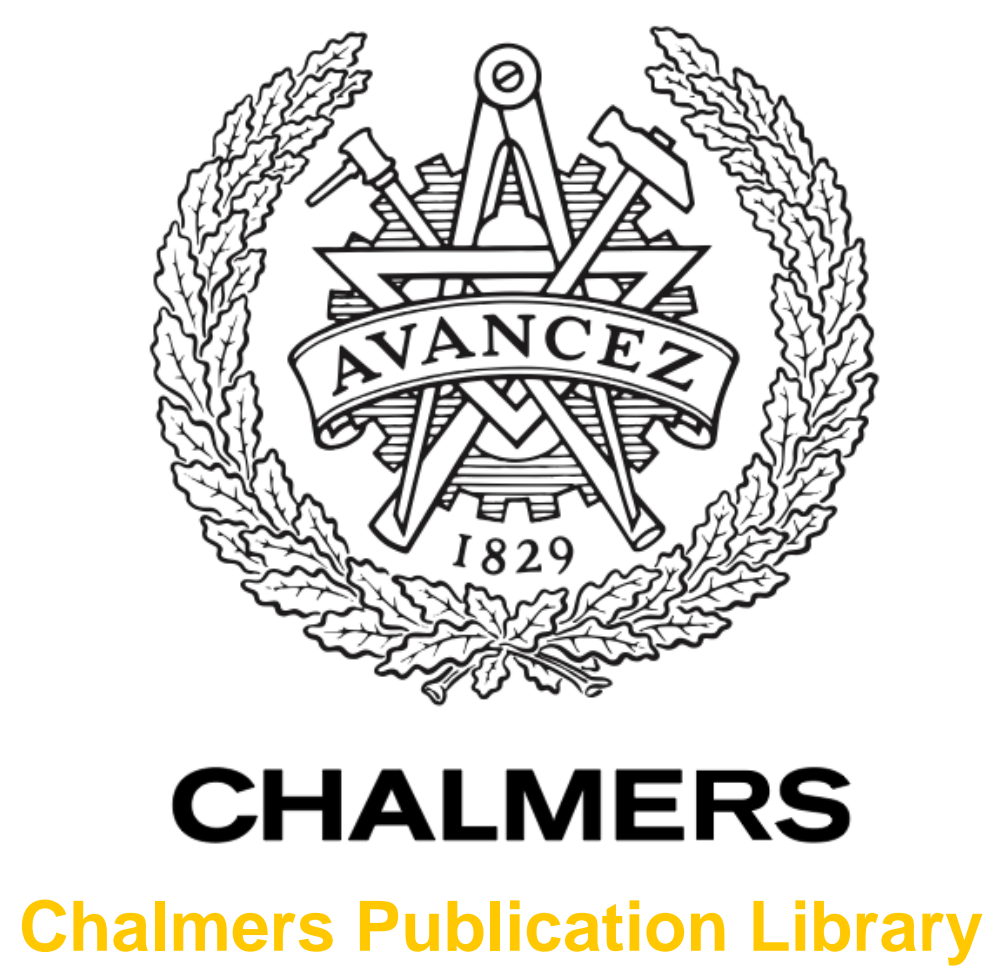

Elastic wave scattering by a rectangular crack near a non-planar back surface

This document has been downloaded from Chalmers Publication Library (CPL). It is the author's version of a work that was accepted for publication in:

Engineering analysis with boundary elements (ISSN: 0955-7997)

Citation for the published paper:

Westlund, J. ; Boström, A. (2012) "Elastic wave scattering by a rectangular crack near a nonplanar back surface". Engineering analysis with boundary elements, vol. 36 pp. 1189-1198.

http://dx.doi.org/10.1016/j.enganabound.2012.02.01

Down「oaded from: http://publications.lib.chalmers.se/publication/168004

Notice: Changes introduced as a result of publishing processes such as copy-editing and formatting may not be reflected in this document. For a definitive version of this work, please refer to the published source. Please note that access to the published version might require a subscription. 


\title{
Elastic wave scattering by a rectangular crack near a non-planar back surface
}

\author{
J. Westlund, A. Boström \\ Chalmers University of Technology, Department of Applied Mechanics, SE-412 96 \\ Gothenburg, Sweden
}

\begin{abstract}
A 3D model of nondestructive ultrasonic testing for cracks near a non-planar back surface is presented. The scattering by an interior rectangular crack in a thick-walled component with a back surface of general geometry is considered. The 3D wave scattering problem is solved using boundary integral equation methods (BIEMs): the boundary element method (BEM) for the back surface displacement is combined with an analytical technique for the hypersingular traction boundary integral equation for the crack opening displacement. The solution method generates many unknowns, but by applying a threshold criterion a sparse approximation of the system matrix is obtained such that a fast sparse solver may be used. The computations are accelerated further by using the stationary phase approximation for the computation of probe field integrals. The action of ultrasonic probes in transmission and reception, calibration by side-drilled holes and effects of material damping are taken into account in the model, and a few numerical examples illustrate the influence of the back surface geometry.
\end{abstract}

Keywords: Elastic waves, Boundary integral equation method, Boundary element method, Scattering, Nondestructive testing, Ultrasonics

\section{Introduction}

Quantitative methods of nondestructive evaluation (NDE) today play an important role in in-service inspection and maintenance of structures, with especially important applications in the aerospace and nuclear industries where failures can have very severe consequences. Among the several methods of NDE that exist today the ultrasonic techniques, the focus of the present paper, are arguably the most important.

As is recognized by Thompson and Gray [1] and Achenbach [2, 3, 4], the need of a measurement model - a model of the complete testing situation - is common

Email address: anders.bostrom@chalmers.se (A. Boström) 
to all methods of NDE. In the case of ultrasonic testing such a model should include the generation of the incoming ultrasound by the transmitting probe, the interaction of this wave field with the defect, and the signal response obtained as output at the receiving probe due to excitation by the wave field scattered by the defect. Access to a good measurement model has major benefits: it enables parametric studies such that costly and time-consuming experiments can be avoided, thus making the model an important tool when designing and optimizing testing procedures. A model can also be a very valuable tool in the interpretation of experimental data, thus increasing the physical understanding of the wave propagation. Models are also very useful in the assessment of probability of detection of defects.

In this paper a model of ultrasonic testing for cracks near a non-planar back surface is presented. The application in mind is the testing of nuclear power plant components such as thick-walled pipes, which can feature diameter changes or connections with complex, non-planar geometries. The presence of these non-planar back surfaces complicates the testing since the signal from a defect may be strongly influenced or even masked by the signal from the nonplanar surface.

The solution method employed in the model is based on boundary integral equation methods, BIEMs, with the major advantage of being essentially exact methods such that the results are valid both for low, intermediate and high frequencies. This is in contrast to the approximate theories such as the Geometrical Theory of Diffraction (GTD) and the elastodynamic Kirchhoff theory used, for example, in the models by Calmon et al. [5] and Chapman [6]. These approximate theories provide powerful and efficient methods with the possibility to treat complex geometries, but finding the bounds of applicability of the theories in a specific case can be difficult. The identification of such bounds may actually be an important application of an essentially exact model, as discussed in the paper by Schafbuch et al. [7] who employ the boundary element method (BEM) in a model of ultrasonic NDE. The authors also make comparisons of their BEM-results with the results of approximate, asymptotic theories and also experiments.

This paper is organized as follows. In Section 2 the problem formulation is given, and in Section 3 the reformulation of the wave scattering problem as two coupled boundary integral equations, BIEs, is described. In Section 4 the action of ultrasonic probes in transmission and reception is modeled, while the discretization and the numerical solution method are described in Section 5. Finally, in Section 6, the numerical computations are discussed and in Section 7 a few numerical examples are given.

\section{Problem formulation}

The scattering geometry is depicted in Fig. 1, where an interior rectangular crack with sides $2 a_{1}$ and $2 a_{2}$ is located in a thick-walled component with a nonplanar back surface. Conventional transmitting and receiving ultrasonic contact probes $(\mathrm{T})$ and $(\mathrm{R})$, respectively, scan along the surface of the component. The 
same probe may also be used in both transmission and reception, in the common case of pulse-echo testing.

The figure also introduces four coordinate systems: the crack coordinate system $\boldsymbol{x}^{\mathrm{c}}$, the back surface system $\boldsymbol{x}^{\mathrm{b}}$, the transmitter system $\boldsymbol{x}^{\mathrm{t}}$ and the receiver system $\boldsymbol{x}^{\mathrm{r}}$. The superscripts ' $c$ ', ' $\mathrm{b}$ ', ' $\mathrm{t}$ ' and ' $\mathrm{r}$ ' on quantities indicate that they are represented in the corresponding coordinate system. Omitted superscripts indicate expressions which hold in any of the coordinate systems.

The location of the crack center relative to the back surface coordinate system is given by the vector $\boldsymbol{d}$, and the crack orientation relative to the back surface coordinate system is described by the three Euler angles $\left(\varphi^{\mathrm{c}}, \theta^{\mathrm{c}}, \psi^{\mathrm{c}}\right)$. Here $\varphi^{\mathrm{c}}$ is the rotation around the $x_{3}^{\mathrm{b}}$-axis, $\theta^{\mathrm{c}}$ the rotation around the intermediate $x_{2}$-axis and $\psi^{\mathrm{c}}$ the rotation around the $x_{3}^{\mathrm{c}}$-axis. The standard transformation rules for the transformation between the coordinate systems apply, with the transformation (rotation) matrix $\mathrm{R}^{\mathrm{c}}$ from $\boldsymbol{x}^{\mathrm{b}}$ to $\boldsymbol{x}^{\mathrm{c}}$. The positions of the transmitting and receiving probes relative to the back surface coordinate system are given by the vectors $\boldsymbol{d}^{\mathrm{T}}$ and $\boldsymbol{d}^{\mathrm{R}}$, respectively, and the probe systems and the back surface system are assumed to be collinear.

The shape of the back surface is described by the function $g\left(x_{1}^{\mathrm{b}}, x_{2}^{\mathrm{b}}\right)$ and is allowed to be quite arbitrary, as long as it has a continuous normal vector. In the subsequent numerical solution procedure the infinite back surface is truncated and discretized, and $\left(T_{1}, T_{2}\right)$ and $\left(T_{3}, T_{4}\right)$ denote the corresponding lower and upper truncation limits in the $x_{1}^{\mathrm{b}}$ - and $x_{2}^{\mathrm{b}}$-directions, respectively.

The component is linearly elastic, isotropic and homogeneous in the exterior of the crack, with Lamé constants $\lambda$ and $\mu$ and density $\rho$. The distance between the crack and the back surface may be arbitrary as long as the crack is interior, but the distance between the scanning surface of the component and the crack and back surface is assumed to be at least a couple of wavelengths. Thus the multiple scattering between the crack and the back surface is fully taken into account, whereas the multiple scattering between the scanning surface and the crack and back surface is neglected. This also means that the incident field from the transmitting probe can be calculated as the field in a half-space.

Only time-harmonic elastodynamics is considered and the time-factor $\mathrm{e}^{-\mathrm{i} \omega t}$, with $\omega$ denoting the angular frequency and $t$ the time, is suppressed throughout. The elastodynamic equation of motion is then:

$$
\nabla \cdot \boldsymbol{\sigma}+\rho \omega^{2} \boldsymbol{u}=\mathbf{0},
$$

where $\boldsymbol{\sigma}$ is the Cauchy stress tensor, $\boldsymbol{u}$ the displacement vector and the constitutive equation relating the stresses and displacement gradients is Hooke's law:

$$
\boldsymbol{\sigma}=\mathcal{C}: \nabla \boldsymbol{u} .
$$

For the isotropic and homogeneous materials considered here the elastic stiffness tensor $\mathcal{C}$ may be expressed as $\mathcal{C}=\lambda \mathbf{I}_{2} \otimes \mathbf{I}_{2}+2 \mu \mathbf{S}_{4}$, where $\mathbf{I}_{2}$ is the second-order identity tensor and $\mathbf{S}_{4}$ the symmetric fourth-order identity tensor. 
The combination of the explicit expression for the stiffness tensor with Hooke's law (2.2) plugged into the equation of motion (2.1) yields the usual elastodynamic wave equation:

$$
k_{\mathrm{p}}^{-2} \nabla(\nabla \cdot \boldsymbol{u})-k_{\mathrm{s}}^{-2} \nabla \times(\nabla \times \boldsymbol{u})+\boldsymbol{u}=\mathbf{0},
$$

where $k_{\mathrm{p}}=\omega / c_{\mathrm{p}}$ is the pressure wave number, $c_{\mathrm{p}}=((\lambda+2 \mu) / \rho)^{1 / 2}$ the pressure wave speed, $k_{\mathrm{s}}=\omega / c_{\mathrm{s}}$ the shear wave number and $c_{\mathrm{s}}=(\mu / \rho)^{1 / 2}$ the shear wave speed. Adding the traction-free boundary conditions on the crack and back surface, a specified incident wave field and the usual outgoing radiation condition at infinity completes the formulation of the wave scattering problem at hand.

\section{The integral equations}

The wave scattering problem is solved using boundary integral equation (BIE) methods; the scattering problem is reformulated as two coupled BIEs which are then solved simultaneously. The BIE-reformulation is based on use of the isotropic free-space outgoing wave Green's tensor, commonly called the Helmholtz fundamental solution and here denoted by $\boldsymbol{U}^{k}(\boldsymbol{x}, \boldsymbol{y} ; \omega)$. It is defined as the outward propagating solution of the equation:

$$
\nabla \cdot \boldsymbol{\Sigma}^{k}(\boldsymbol{x}, \boldsymbol{y} ; \omega)+\rho \omega^{2} \boldsymbol{U}^{k}(\boldsymbol{x}, \boldsymbol{y} ; \omega)=-\delta(\boldsymbol{x}-\boldsymbol{y}) \mathbf{e}_{k},
$$

where the corresponding stress tensor is $\boldsymbol{\Sigma}^{k}(\boldsymbol{x}, \boldsymbol{y} ; \omega) \equiv \mathcal{C}: \nabla \boldsymbol{U}^{k}(\boldsymbol{x}, \boldsymbol{y} ; \omega), \delta(\boldsymbol{x}-$ $\boldsymbol{y})$ denotes the 3D Dirac delta distribution and $\mathbf{e}_{k}$ is the unit vector in the $k$ direction, $k=1,2,3$. Throughout the paper, the $\nabla$-operator always acts on the $\boldsymbol{x}$-coordinates.

The Green's tensor may be calculated using the formula (see e.g. Ström [8]):

$$
\begin{aligned}
U_{j}^{k}(\boldsymbol{x}, \boldsymbol{y} ; \omega)=\frac{1}{\mu k_{\mathrm{s}}^{2}}[ & k_{\mathrm{s}}^{2} \delta_{j k} \mathrm{G}\left(\boldsymbol{x}, \boldsymbol{y} ; k_{\mathrm{s}}\right) \\
& \left.+\frac{\partial}{\partial x_{j}} \frac{\partial}{\partial x_{k}}\left(\mathrm{G}\left(\boldsymbol{x}, \boldsymbol{y} ; k_{\mathrm{s}}\right)-\mathrm{G}\left(\boldsymbol{x}, \boldsymbol{y} ; k_{\mathrm{p}}\right)\right)\right],
\end{aligned}
$$

where $\delta_{j k}$ is the Kronecker delta and $\mathrm{G}\left(\boldsymbol{x}, \boldsymbol{y} ; k_{m}\right)$ is the outward-propagating fundamental solution of Helmholtz equation with wave number $k_{m}, m=\mathrm{p}$, s. To obtain the Green's tensor on closed form, Eq. (3.2) is used in conjunction with the standard closed form expression for G:

$$
\mathrm{G}\left(\boldsymbol{x}, \boldsymbol{y} ; k_{m}\right)=\frac{\mathrm{e}^{\mathrm{i} k_{m}|\boldsymbol{x}-\boldsymbol{y}|}}{4 \pi|\boldsymbol{x}-\boldsymbol{y}|} .
$$

\subsection{The back surface integral equation}

The back surface integral equation may be derived directly using the Green's tensor, the divergence theorem and a limiting process. However, in order to avoid strongly singular integrals an indirect regularization approach (see 
Bonnet [9]) is also followed. This approach transfers the singularity of the dynamic Green's stress tensor $\boldsymbol{\Sigma}^{k}(\boldsymbol{x}, \boldsymbol{y} ; \omega)$ to the static Green's stress tensor $\boldsymbol{\Sigma}^{k}(\boldsymbol{x}, \boldsymbol{y})=\mathcal{C}: \nabla \boldsymbol{U}^{k}(\boldsymbol{x}, \boldsymbol{y})$, with $\boldsymbol{U}^{k}(\boldsymbol{x}, \boldsymbol{y})$ denoting the corresponding static displacement tensor. This Green's tensor, also commonly called the Kelvin fundamental solution, is the solution of Eq. (3.1) for $\omega=0$ and is given explicitly by e.g. Bonnet [9]. By transferring the singularity to the static Green's tensor it becomes possible to evaluate the strongly singular integral analytically. The result is a back surface BIE containing no worse than weakly singular integrals, as long as the displacement $\boldsymbol{u}$ satisfies the usual assumption of Höldercontinutity $[9,10]$.

The derivation of the back surface BIE and the regularization is a straightforward extension to 3D of the results in the paper by Westlund and Boström [11], resulting in the following regularized back surface integral equation:

$$
\begin{aligned}
& \int_{S_{T}} u_{i}^{\mathrm{b}}\left(\boldsymbol{x}^{\mathrm{b}}\right)\left[\Sigma_{i j}^{k}\left(\boldsymbol{x}^{\mathrm{b}}, \boldsymbol{y}^{\mathrm{b}} ; \omega\right)-\Sigma_{i j}^{k}\left(\boldsymbol{x}^{\mathrm{b}}, \boldsymbol{y}^{\mathrm{b}}\right)\right] \mathrm{n}_{j}^{\mathrm{b}}\left(\boldsymbol{x}^{\mathrm{b}}\right) \mathrm{d} S_{\boldsymbol{x}^{\mathrm{b}}} \\
+ & \int_{S_{T}}\left[u_{i}^{\mathrm{b}}\left(\boldsymbol{x}^{\mathrm{b}}\right)-u_{i}^{\mathrm{b}}\left(\boldsymbol{y}^{\mathrm{b}}\right)\right] \Sigma_{i j}^{k}\left(\boldsymbol{x}^{\mathrm{b}}, \boldsymbol{y}^{\mathrm{b}}\right) \mathrm{n}_{j}^{\mathrm{b}}\left(\boldsymbol{x}^{\mathrm{b}}\right) \mathrm{d} S_{\boldsymbol{x}^{\mathrm{b}}} \\
+ & \mathrm{R}_{i p}^{\mathrm{c}} \mathrm{R}_{3 q}^{\mathrm{c}} \int_{-a_{2}}^{a_{2}} \int_{-a_{1}}^{a_{1}} \Delta u_{i}^{\mathrm{c}}\left(x_{1}^{\mathrm{c}}, x_{2}^{\mathrm{c}}\right) \Sigma_{p q}^{k}\left(\left(\mathrm{R}^{\mathrm{c}}\right)^{\mathrm{T}}\left\{x_{1}^{\mathrm{c}}, x_{2}^{\mathrm{c}}, 0\right\}+\boldsymbol{d}^{\mathrm{b}}, \boldsymbol{y}^{\mathrm{b}} ; \omega\right) \mathrm{d} x_{1}^{\mathrm{c}} \mathrm{d} x_{2}^{\mathrm{c}} \\
- & \frac{1}{2} u_{k}^{\mathrm{b}}\left(\boldsymbol{y}^{\mathrm{b}}\right)+u_{k}^{\mathrm{in}, \mathrm{b}}\left(\boldsymbol{y}^{\mathrm{b}}\right)=0,
\end{aligned}
$$

where $\boldsymbol{y} \in S_{T}, \boldsymbol{u}$ is the total displacement field, $\mathbf{n}$ the upward unit normal vector of the back surface, $\Delta \boldsymbol{u}$ the crack opening displacement (COD): $\Delta \boldsymbol{u}\left(x_{1}^{\mathrm{c}}, x_{2}^{\mathrm{c}}\right) \equiv \boldsymbol{u}\left(x_{1}^{\mathrm{c}}, x_{2}^{\mathrm{c}}, 0^{+}\right)-\boldsymbol{u}\left(x_{1}^{\mathrm{c}}, x_{2}^{\mathrm{c}}, 0^{-}\right), \boldsymbol{u}^{\mathrm{in}}$ the incident displacement field from the transmitting probe and $k=1,2,3$. In this integral equation the integrals over the infinite back surface have been replaced by integrals over the truncated back surface $S_{T}$. This approximation is expected to be valid for sufficiently large truncation limits $\left(T_{1}, T_{2}\right)$ and $\left(T_{3}, T_{4}\right)$.

\subsection{The crack integral equation}

An integral representation for the displacement field may be derived using the divergence theorem and the Green's tensor, just as is done in the derivation of the back surface integral equation above and also discussed by Pao and Varatharajulu [12]. The result is the following equation, valid for interior points $\boldsymbol{y}$ :

$$
\begin{aligned}
& u_{k}^{\mathrm{c}}\left(\boldsymbol{y}^{\mathrm{c}}\right)=\int_{S_{T}} u_{i}^{\mathrm{c}}\left(\boldsymbol{x}^{\mathrm{c}}\right) \Sigma_{i j}^{k}\left(\boldsymbol{x}^{\mathrm{c}}, \boldsymbol{y}^{\mathrm{c}} ; \omega\right) \mathrm{n}_{j}^{\mathrm{c}}\left(\boldsymbol{x}^{\mathrm{c}}\right) \mathrm{d} S_{\boldsymbol{x}^{\mathrm{c}}} \\
& +\int_{-a_{2}}^{a_{2}} \int_{-a_{1}}^{a_{1}} \Delta u_{i}^{\mathrm{c}}\left(x_{1}^{\mathrm{c}}, x_{2}^{\mathrm{c}}\right) \Sigma_{i 3}^{k}\left(\left\{x_{1}^{\mathrm{c}}, x_{2}^{\mathrm{c}}, 0\right\}, \boldsymbol{y}^{\mathrm{c}} ; \omega\right) \mathrm{d} x_{1}^{\mathrm{c}} \mathrm{d} x_{2}^{\mathrm{c}}+u_{k}^{\mathrm{in}, \mathrm{c}}\left(\boldsymbol{y}^{\mathrm{c}}\right) .
\end{aligned}
$$

It is well-known that displacement BIEs degenerate for cracks [9, 13], so for the crack a traction BIE is used instead. The crack integral equation is then derived 
from the integral representation by applying the stress operator, letting the source point approach the crack (from either side) and invoking the boundary condition to obtain:

$$
\begin{array}{r}
\lim _{y_{3}^{\mathrm{c}} \rightarrow 0} \sigma_{i 3}^{\mathrm{c}}\left(y_{1}^{\mathrm{c}}, y_{2}^{\mathrm{c}}, y_{3}^{\mathrm{c}}\right)=\int_{S_{T}} u_{m}^{\mathrm{c}}\left(\boldsymbol{x}^{\mathrm{c}}\right) \mathcal{C}_{i 3 k l} \frac{\partial}{\partial y_{l}^{\mathrm{c}}} \Sigma_{m n}^{k}\left(\boldsymbol{x}^{\mathrm{c}},\left\{y_{1}^{\mathrm{c}}, y_{2}^{\mathrm{c}}, 0\right\} ; \omega\right) \mathrm{n}_{n}^{\mathrm{c}}\left(\boldsymbol{x}^{\mathrm{c}}\right) \mathrm{d} S_{\boldsymbol{x}^{\mathrm{c}}} \\
+\lim _{y_{3}^{\mathrm{c}} \rightarrow 0} \int_{-a_{2}}^{a_{2}} \int_{-a_{1}}^{a_{1}} \Delta u_{m}^{\mathrm{c}}\left(x_{1}^{\mathrm{c}}, x_{2}^{\mathrm{c}}\right) \mathcal{C}_{i 3 k l} \\
\frac{\partial}{\partial y_{l}^{\mathrm{c}}} \Sigma_{m 3}^{k}\left(\left\{x_{1}^{\mathrm{c}}, x_{2}^{\mathrm{c}}, 0\right\}, \boldsymbol{y}^{\mathrm{c}} ; \omega\right) \mathrm{d} x_{1}^{\mathrm{c}} \mathrm{d} x_{2}^{\mathrm{c}} \\
+\mathcal{C}_{i 3 k l} \frac{\partial}{\partial y_{l}^{\mathrm{c}}} u_{k}^{\mathrm{in}, \mathrm{c}}\left(\left\{y_{1}^{\mathrm{c}}, y_{2}^{\mathrm{c}}, 0\right\}\right)=0, \quad(3.6)
\end{array}
$$

where $i=1,2,3,\left|y_{1}^{\mathrm{c}}\right|<a_{1},\left|y_{2}^{\mathrm{c}}\right|<a_{2}$ and $\mathcal{C}_{i j k l}$ are the components of the elastic stiffness tensor $\mathcal{C}$. The second integral in this equation is of the hypersingular type (see e.g. Refs. $[13,14,15,16]$ for discussions of hypersingular integrals), so the limit cannot be moved inside the integral. However, the present solution method enables an explicit evaluation of the limit at a later stage, as described in section 5 .

\section{The ultrasonic probes}

This paper considers conventional ultrasonic contact probes consisting of a piezoelectric crystal attached to a plastic wedge, with a couplant usually applied between the component and the probe. In order to account for the action of transmitting ultrasonic probes of this type, the probe model developed by Boström and Wirdelius [17] is used. This model is based on prescribing the traction on the scanning surface of the component, and the traction is taken as that due to a plane $\mathrm{SH}, \mathrm{SV}$ or $\mathrm{P}$ wave with given amplitude and propagation direction. For the transmitting probe (T), the boundary condition on the upper surface of the component is taken as the traction:

$$
\begin{aligned}
& \boldsymbol{t}^{\mathrm{t}}= \\
& \left\{\begin{array}{l}
\mathrm{i} A_{0} f \mu k_{\mathrm{p}}\left[\delta \sin 2 \gamma_{\mathrm{t}} \mathbf{e}_{x_{1}^{\mathrm{t}}}+\left(\frac{k_{\mathrm{s}}^{2}}{k_{\mathrm{p}}^{2}}-2 \sin ^{2} \gamma_{\mathrm{t}}\right) \mathbf{e}_{x_{3}^{\mathrm{t}}}\right] \mathrm{e}^{-\mathrm{i} k_{\mathrm{p}} x_{1}^{\mathrm{t}} \sin \gamma_{\mathrm{t}}}, \mathrm{P} \text { probe, } \\
\mathrm{i} A_{0} f \mu k_{\mathrm{s}}\left[-\delta \cos 2 \gamma_{\mathrm{t}} \mathbf{e}_{x_{1}^{\mathrm{t}}}+\sin 2 \gamma_{\mathrm{t}} \mathbf{e}_{x_{3}^{\mathrm{t}}}\right] \mathrm{e}^{-\mathrm{i} k_{\mathrm{s}} x_{1}^{\mathrm{t}} \sin \gamma_{\mathrm{t}}}, \mathrm{SV} \text { probe, } \\
\mathrm{i} A_{0} f \mu k_{\mathrm{s}} \delta \cos \gamma_{\mathrm{t}} \mathbf{e}_{x_{2}^{\mathrm{t}}} \mathrm{e}^{-\mathrm{i} k_{\mathrm{s}} x_{1}^{\mathrm{t}} \sin \gamma_{\mathrm{t}}}, \mathrm{SH} \text { probe, }
\end{array}\right.
\end{aligned}
$$

beneath the surface of the probe and $\boldsymbol{t}^{\mathrm{t}}=\mathbf{0}$ elsewhere. The surface of the probe is assumed to be either elliptical or rectangular, and the function $f$ is introduced in order to allow for a non-constant traction tapering off towards the edges of the probe. The transmitting probe angle $\gamma_{\mathrm{t}}$ is the rotation in the $x_{2}^{\mathrm{t}}$-direction measured from the negative $x_{3}^{\mathrm{t}}$-axis, the parameter $\delta$ (with $0 \leq \delta \leq 1$ ) accounts 
for the effect of a couplant applied between the probe and the scanning surface and $A_{0}$ is the amplitude.

When multiple reflections between the scanning surface and the crack and back surface are neglected, the action of the probe can be calculated as in a halfspace and enables a solution of the boundary value problem for the incident field using a double Fourier transform. With the notation of Boström et al. [18] for the vector plane waves, the Fourier expansion reads:

$$
\boldsymbol{u}^{\mathrm{in}, \mathrm{t}}\left(\boldsymbol{x}^{\mathrm{t}}\right)=\sum_{j=1}^{3} \int_{C_{-}} \xi_{j}(\alpha, \beta) \boldsymbol{\varphi}_{j}^{\mathrm{t}}\left(\alpha, \beta ; \boldsymbol{x}^{\mathrm{t}}\right) \mathrm{d} \hat{\boldsymbol{\gamma}},
$$

where $\alpha$ and $\beta$ are the spherical angles of propagation of the plane waves, $\boldsymbol{\varphi}_{1}$ is the $\mathrm{SH}$-wave, $\varphi_{2}$ the $\mathrm{SV}$-wave and $\varphi_{3}$ the $\mathrm{P}$-wave. The functions $\xi_{j}$ are determined by converting Eq. (4.2) to rectangular coordinates $(q, p)$ in Fourier space, computing the corresponding traction and identifying with the Fourier transform $\boldsymbol{T}^{\mathrm{t}}$ of the prescribed traction $\boldsymbol{t}^{\mathrm{t}}$ given by Eq. (4.1). The details are given in the paper by Boström and Wirdelius [17], where the vector plane waves $\boldsymbol{\varphi}_{j}$, the functions $\xi_{j}$, the integration contour $C_{-}$, the integration measure $\mathrm{d} \hat{\gamma}$ and the vector $\boldsymbol{T}^{\mathrm{t}}$ are all listed explicitly.

In order to predict the signal response measured in an ultrasonic testing situation, the action of the receiving probe must be modeled. This is achieved by using the electromechanical reciprocity relation by Auld [19], which states that the change in the received signal induced by the presence of a defect may be computed by considering the two states (1): the transmitting probe illuminating the medium with the defect present and (2): the receiving probe acting as a transmitter with the defect absent. Applied to this case Auld's reciprocity relation gives the crack signal response (which includes multiple reflections between the crack and back surface) as:

$$
\delta \Gamma_{C}=\frac{-\mathrm{i} \omega}{4 P} \int_{-a_{2}}^{a_{2}} \int_{-a_{1}}^{a_{1}} \Delta u_{i}^{\mathrm{c}}\left(x_{1}^{\mathrm{c}}, x_{2}^{\mathrm{c}}\right) \sigma_{i 3}^{\mathrm{re}, \mathrm{c}}\left(x_{1}^{\mathrm{c}}, x_{2}^{\mathrm{c}}, 0\right) \mathrm{d} x_{1}^{\mathrm{c}} \mathrm{d} x_{2}^{\mathrm{c}},
$$

and the back surface signal response (in the absence of the crack) as:

$$
\delta \Gamma_{B S}=\frac{-\mathrm{i} \omega}{4 P} \int_{S_{T}} u_{i}^{\mathrm{re}, \mathrm{b}}\left(\boldsymbol{x}^{\mathrm{b}}\right) \sigma_{i j}^{\mathrm{in}, \mathrm{b}}\left(\boldsymbol{x}^{\mathrm{b}}\right) \mathrm{n}_{j}^{\mathrm{b}}\left(\boldsymbol{x}^{\mathrm{b}}\right) \mathrm{d} S_{\boldsymbol{x}^{\mathrm{b}}},
$$

such that the total signal response is $\delta \Gamma_{T o t}=\delta \Gamma_{B S}+\delta \Gamma_{C}$. In the above equations the superscript 're' denotes quantities computed with the back surface present but the crack absent and the incident field generated by the receiving probe acting as transmitter, and the superscript 'in' denotes quantities computed with both back surface and crack absent and the incident field generated by the transmitting probe. The COD $\Delta u_{i}$ is due to an incident field from the transmitting probe with both crack and back surface present. The probes are transmitting at the fixed angular frequency $\omega$, and the quantity $P$ is essentially the power supplied to the transmitting probe. For detailed discussions of probe signal response calculations based on Auld's reciprocity relation the reader is referred to the papers by Thompson [20] and Mattsson and Niklasson [21]. 


\section{Solution of the integral equations}

The two coupled boundary integral equations (3.4) and (3.6) are solved by discretizing them and subsequently solving the resulting system of linear algebraic equations. As is always the case in BIEMs, the presence of singular integrals requires special attention. However, due to the regularization approach followed for the back surface BIE, Eq. (3.4), it contains no strong singularities and is immediately suited for a standard boundary element discretization. Use of the BEM for the back surface has the major benefit of allowing for a general shape of the back surface.

The hypersingular integral in the crack BIE, Eq. (3.6), also requires some kind of regularization. One way of achieving this is to use the Galerkin method, in which the necessary regularization is achieved automatically by the repeated integration over the crack. However, the Galerkin method typically leads to more expensive computations compared to other regularization techniques which are used in conjunction with the collocation method. Exceptions are found for simple defect types like the rectangular crack considered in this paper, for which a suitable Fourier series expansion of the COD in Chebyshev functions (see e.g. Refs. [22, 23]) enables an analytical evaluation of the crack integrals occurring in the Galerkin method. The result is a computationally efficient solution procedure for the crack BIE which automatically resolves the difficulties associated with hypersingular integrals.

The Chebyshev functions used in the series expansion of the COD are defined by:

$$
\psi_{n}(v)= \begin{cases}\frac{1}{\pi} \cos (n \arcsin v), & n=1,3, \ldots, \\ \frac{1}{\pi} \sin (n \arcsin v), & n=2,4, \ldots,\end{cases}
$$

such that the expansion reads:

$$
\Delta u_{i}^{\mathrm{c}}\left(x_{1}^{\mathrm{c}}, x_{2}^{\mathrm{c}}\right)=\sum_{n_{1}=1}^{N_{1}} \sum_{n_{2}=1}^{N_{2}} \alpha_{i n_{1} n_{2}} \psi_{n_{1}}\left(x_{1}^{\mathrm{c}} / a_{1}\right) \psi_{n_{2}}\left(x_{2}^{\mathrm{c}} / a_{2}\right) .
$$

This expansion allows for an analytical evaluation of the crack integrals arising in the crack BIE, due to the fact that the Chebyshev functions satisfy the integral relation:

$$
\int_{-1}^{1} \psi_{n}(v) \mathrm{e}^{-\mathrm{i} \gamma v} \mathrm{~d} v=\frac{n}{\gamma} \mathrm{J}_{n}(\gamma)
$$

where $\mathrm{J}_{n}$ is the Bessel function of the first kind and order $n$. The expansion also exhibits the correct behavior at the crack edges since the Chebyshev functions $\psi_{n}(v)$ are of the form of a polynomial of degree $n-1$ multiplied by the factor $\sqrt{1-v^{2}}$. At the corners of the rectangular crack, on the other hand, slightly stronger singularities are expected [24], but since this behavior is difficult to incorporate in the expansion no measure is taken to include these singularities. In addition, the results of Nishimura and Kobayashi [25] show that neglecting 
the corner singularities has no significant influence on the results; they report accurate results using a discretization which exhibits the correct behavior at the crack edges but is actually completely non-singular at the crack corners.

A standard boundary element discretization (see e.g. Refs. $[9,26,27]$ ) of the back surface is performed using nine-noded quadrilateral elements and isoparametrical Lagrangian interpolation. With $N_{\text {node }}$ denoting the number of nodes on the truncated back surface $S_{T}$ after it has been discretized into $N_{e}$ elements, the number of unknowns on the back surface is $3 N_{\text {node }}$. The discretization of the COD given by Eq. (5.1) results in $3 N_{1} N_{2}$ unknown expansion coefficients $\alpha_{i n_{1} n_{2}}$, such that the total number of unknowns is $N_{\text {dof }}=3\left(N_{\text {node }}+N_{1} N_{2}\right)$. The same number of equations is obtained by collocating the back surface BIE at each node point $\boldsymbol{y}_{n_{c}}$ on $S_{T}$ and by using the Galerkin method for the crack BIE: the BIE is projected on the $N_{1} N_{2}$ expansion functions.

Letting $\left\{E_{e}\right\}_{e=1}^{N_{e}}$ denote the boundary elements, the partly discretized back surface BIE can be written:

$$
\begin{aligned}
& \sum_{e=1}^{N_{e}}\left[\int_{E_{e}} u_{i}^{\mathrm{b}}\left(\boldsymbol{x}^{\mathrm{b}}\right)\left[\Sigma_{i j}^{k}\left(\boldsymbol{x}^{\mathrm{b}}, \boldsymbol{y}_{n_{c}}^{\mathrm{b}} ; \omega\right)-\Sigma_{i j}^{k}\left(\boldsymbol{x}^{\mathrm{b}}, \boldsymbol{y}_{n_{c}}^{\mathrm{b}}\right)\right] \mathrm{n}_{j}^{\mathrm{b}}\left(\boldsymbol{x}^{\mathrm{b}}\right) \mathrm{d} S_{\boldsymbol{x}^{\mathrm{b}}}\right. \\
& \left.\quad+\int_{E_{e}}\left[u_{i}^{\mathrm{b}}\left(\boldsymbol{x}^{\mathrm{b}}\right)-u_{i}^{\mathrm{b}}\left(\boldsymbol{y}_{n_{c}}^{\mathrm{b}}\right)\right] \Sigma_{i j}^{k}\left(\boldsymbol{x}^{\mathrm{b}}, \boldsymbol{y}_{n_{c}}^{\mathrm{b}}\right) \mathrm{n}_{j}^{\mathrm{b}}\left(\boldsymbol{x}^{\mathrm{b}}\right) \mathrm{d} S_{\boldsymbol{x}^{\mathrm{b}}}\right] \\
& \quad+\mathrm{R}_{i p}^{\mathrm{c}} \mathrm{R}_{3 q}^{\mathrm{c}} \sum_{n_{1}=1}^{N_{1}} \sum_{n_{2}=1}^{N_{2}} \alpha_{i n_{1} n_{2}} \int_{-a_{2}}^{a_{2}} \int_{-a_{1}}^{a_{1}} \psi_{n_{2}}\left(x_{2}^{\mathrm{c}} / a_{2}\right) \psi_{n_{1}}\left(x_{1}^{\mathrm{c}} / a_{1}\right) \\
& \times \Sigma_{p q}^{k}\left(\left(\mathrm{R}^{\mathrm{c}}\right)^{\mathrm{T}}\left\{x_{1}^{\mathrm{c}}, x_{2}^{\mathrm{c}}, 0\right\}+\boldsymbol{d}^{\mathrm{b}}, \boldsymbol{y}_{n_{c}}^{\mathrm{b}} ; \omega\right) \mathrm{d} x_{1}^{\mathrm{c}} \mathrm{d} x_{2}^{\mathrm{c}} \\
& -\frac{1}{2} u_{k}^{\mathrm{b}}\left(\boldsymbol{y}_{n_{c}}^{\mathrm{b}}\right)+u_{k}^{\mathrm{in}, \mathrm{b}}\left(\boldsymbol{y}_{n_{c}}^{\mathrm{b}}\right)=0, \text { for } n_{c}=1,2, \ldots, N_{n o d e} \text { and } k=1,2,3 .
\end{aligned}
$$


Further, the partly discretized crack BIE can be written:

$$
\begin{aligned}
& \sum_{e=1}^{N_{e}} \int_{-a_{2}}^{a_{2}} \int_{-a_{1}}^{a_{1}} \psi_{n_{2}^{\prime}}\left(y_{2}^{\mathrm{c}} / a_{2}\right) \psi_{n_{1}^{\prime}}\left(y_{1}^{\mathrm{c}} / a_{1}\right) \\
& \quad \times\left(\int_{E_{e}} u_{m}^{\mathrm{c}}\left(\boldsymbol{x}^{\mathrm{c}}\right) \mathcal{C}_{i 3 k l} \frac{\partial}{\partial y_{l}^{\mathrm{c}}} \Sigma_{m n}^{k}\left(\boldsymbol{x}^{\mathrm{c}},\left\{y_{1}^{\mathrm{c}}, y_{2}^{\mathrm{c}}, 0\right\} ; \omega\right) \mathrm{n}_{n}^{\mathrm{c}}\left(\boldsymbol{x}^{\mathrm{c}}\right) \mathrm{d} S_{\boldsymbol{x}^{\mathrm{c}}}\right) \mathrm{d} y_{1}^{\mathrm{c}} \mathrm{d} y_{2}^{\mathrm{c}} \\
& +\sum_{n_{1}=1}^{N_{1}} \sum_{n_{2}=1}^{N_{2}} \alpha_{m n_{1} n_{2}} \lim _{y_{3}^{\mathrm{c}} \rightarrow 0} \int_{-a_{2}}^{a_{2}} \int_{-a_{1}}^{a_{1}} \psi_{n_{2}^{\prime}}\left(y_{2}^{\mathrm{c}} / a_{2}\right) \psi_{n_{1}^{\prime}}\left(y_{1}^{\mathrm{c}} / a_{1}\right) \\
& \quad \times\left(\int_{-a_{2}}^{a_{2}} \int_{-a_{1}}^{a_{1}} \psi_{n_{2}}\left(x_{2}^{\mathrm{c}} / a_{2}\right) \psi_{n_{1}}\left(x_{1}^{\mathrm{c}} / a_{1}\right)\right. \\
& \left.\quad \times \mathcal{C}_{i 3 k l} \frac{\partial}{\partial y_{l}^{\mathrm{c}}} \Sigma_{m 3}^{k}\left(\left\{x_{1}^{\mathrm{c}}, x_{2}^{\mathrm{c}}, 0\right\}, \boldsymbol{y}^{\mathrm{c}} ; \omega\right) \mathrm{d} x_{1}^{\mathrm{c}} \mathrm{d} x_{2}^{\mathrm{c}}\right) \mathrm{d} y_{1}^{\mathrm{c}} \mathrm{d} y_{2}^{\mathrm{c}} \\
& +\int_{-a_{2}}^{a_{2}} \int_{-a_{1}}^{a_{1}} \psi_{n_{2}^{\prime}}\left(y_{2}^{\mathrm{c}} / a_{2}\right) \psi_{n_{1}^{\prime}}\left(y_{1}^{\mathrm{c}} / a_{1}\right) \mathcal{C}_{i 3 k l} \frac{\partial}{\partial y_{l}^{\mathrm{c}}} u_{k}^{\mathrm{in}, \mathrm{c}}\left(\left\{y_{1}^{\mathrm{c}}, y_{2}^{\mathrm{c}}, 0\right\}\right) \mathrm{d} y_{1}^{\mathrm{c}} \mathrm{d} y_{2}^{\mathrm{c}}=0, \\
& \text { for } n_{1}^{\prime}=1,2, \ldots, N_{1}, n_{2}^{\prime}=1,2, \ldots, N_{2} \text { and } i=1,2,3 . \quad(5.4)
\end{aligned}
$$

The fully discretized form of the integral equations is obtained after introducing the elementwise representation of the geometry and the unknowns, in terms of the interpolation functions and the node connectivity table, into Eqs. (5.3) and (5.4). Since the procedure is standard the details are omitted. To solve the wave scattering problem it remains to evaluate all boundary element and crack integrals numerically, assemble the system of linear algebraic equations and solve for the unknown back surface node displacements and COD expansion coefficients.

\section{Numerical considerations}

In this section the numerical computations are discussed.

Except for the temporal Fourier transform which is computed using the trapezoidal rule, all numerical integration is performed using ordinary GaussLegendre quadrature. However, due to the presence of singular integrals in the integral equations some special attention is required for accurate numerical integration.

Due to the fact that the back surface BIE (5.3) has been regularized these numerical computations are quite straightforward. However, accurate evaluation of the singular element integrals (element integrals with the collocation point located on the element) still requires some attention. In this paper the integration technique described by Bonnet [9] is followed, in which polar coordinates in the parent element (with the origin at the collocation point) are introduced. For the weakly singular integrand a reformulation using modified shape functions is then used, such that the regularizing effect of the factor $u_{i}^{\mathrm{b}}\left(\boldsymbol{x}^{\mathrm{b}}\right)-u_{i}^{\mathrm{b}}\left(\boldsymbol{y}_{n_{c}}^{\mathrm{b}}\right)$ is exploited fully. An element subdivision into triangles, as 
described by Rezayat et al. [28], followed by a final coordinate transformation back to rectangular coordinates is then used, after which regular Gauss-Legendre quadrature is applied to the resulting completely nonsingular integrals. It may be remarked that the isoparametrical interpolation used ensures that the condition of Hölder-continuity assumed in the derivation of the back surface BIE is fulfilled.

The integrals containing the factor $\Sigma_{i j}^{k}\left(\boldsymbol{x}^{\mathrm{b}}, \boldsymbol{y}_{n_{c}}^{\mathrm{b}} ; \omega\right)-\Sigma_{i j}^{k}\left(\boldsymbol{x}^{\mathrm{b}}, \boldsymbol{y}_{n_{c}}^{\mathrm{b}}\right)$ are nonsingular as they stand and thus require little extra attention. However, to avoid numerical errors a series expansion of the dynamic Green's tensor is used for small distances between the collocation and integration points, such that the singular term is canceled explicitly.

As long as the crack is interior the crack integrals in the back surface BIE (5.3) are regular, and for the examples in this paper 15 Gauss points in each crack direction have proven sufficient. The same holds true for the back surface integrals in the crack BIE (5.4), for which 3 Gauss points in each boundary element direction and 15 Gauss points in each crack direction, respectively, have proven to be sufficient for the examples considered here. However, the number of Gauss points required for these integrations depends strongly on the distance between the crack and back surface such that cracks closer to the back surface require more Gauss points. This matter is discussed in more detail in the paper by Westlund [29] treating a scalar 2D case with the same method of solution.

In order to make use of the integral relation (5.2) when evaluating the incident probe field and crack integrals in Eq. (5.4), the Fourier integral form of the Green's tensor must be used. This form may be obtained by again employing Eq. (3.2), but now instead with the following Fourier integral representation of $\mathrm{G}\left(\boldsymbol{x}, \boldsymbol{y} ; k_{m}\right)$ :

$$
\mathrm{G}\left(\boldsymbol{x}, \boldsymbol{y} ; k_{m}\right)=\frac{\mathrm{i}}{8 \pi^{2}} \int_{-\infty}^{\infty} \int_{-\infty}^{\infty} \frac{1}{h_{m}} \mathrm{e}^{\mathrm{i}\left(q\left(x_{1}-y_{1}\right)+p\left(x_{2}-y_{2}\right)+h_{m}\left|x_{3}-y_{3}\right|\right)} \mathrm{d} q \mathrm{~d} p,
$$

where $h_{m}=h_{m}(q, p)=\left(k_{m}^{2}-q^{2}-p^{2}\right)^{1 / 2}, m=\mathrm{p}, \mathrm{s}$ and the branch of the complex square root chosen such that $\operatorname{Im} \sqrt{z} \geq 0 \forall z \in \mathbb{C}$. After inserting the Fourier representation of the Green's tensor in Eq. (5.4) and evaluating the crack integrals using the integral relation (5.2), the limit may be evaluated since the resulting integral is now convergent in the ordinary sense. The result is:

$$
\begin{gathered}
\lim _{y_{3}^{\mathrm{c}} \rightarrow 0} \int_{-a_{2}}^{a_{2}} \int_{-a_{1}}^{a_{1}} \psi_{n_{2}^{\prime}}\left(y_{2}^{\mathrm{c}} / a_{2}\right) \psi_{n_{1}^{\prime}}\left(y_{1}^{\mathrm{c}} / a_{1}\right)\left(\int_{-a_{2}}^{a_{2}} \int_{-a_{1}}^{a_{1}} \psi_{n_{2}}\left(x_{2}^{\mathrm{c}} / a_{2}\right) \psi_{n_{1}}\left(x_{1}^{\mathrm{c}} / a_{1}\right)\right. \\
\left.\times \mathcal{C}_{i 3 k l} \frac{\partial}{\partial y_{l}^{\mathrm{c}}} \Sigma_{m 3}^{k}\left(\left\{x_{1}^{\mathrm{c}}, x_{2}^{\mathrm{c}}, 0\right\}, \boldsymbol{y}^{\mathrm{c}} ; \omega\right) \mathrm{d} x_{1}^{\mathrm{c}} \mathrm{d} x_{2}^{\mathrm{c}}\right) \mathrm{d} y_{1}^{\mathrm{c}} \mathrm{d} y_{2}^{\mathrm{c}} \\
=2 \mathrm{i} \mu k_{\mathrm{s}}(-1)^{n_{1}+n_{2}} n_{1} n_{2} n_{1}^{\prime} n_{2}^{\prime} \\
\quad \times \int_{-\infty}^{\infty} \int_{-\infty}^{\infty} S_{i m}(q, p) \mathrm{J}_{n_{1}}\left(a_{1} q\right) \mathrm{J}_{n_{1}^{\prime}}\left(a_{1} q\right) \mathrm{J}_{n_{2}}\left(a_{2} p\right) \mathrm{J}_{n_{2}^{\prime}}\left(a_{2} p\right) \frac{\mathrm{d} q \mathrm{~d} p}{q^{2} p^{2}},
\end{gathered}
$$


where the function $S_{i m}(q, p)$ is:

$$
\left[S_{i m}(q, p)\right]=\frac{1}{16 \pi^{2} k_{\mathrm{s}}^{3} s^{2}}\left(\begin{array}{ccc}
p^{2} k_{\mathrm{s}}^{2} h_{\mathrm{s}}+\frac{q^{2}}{h_{\mathrm{s}}} R & \frac{q p}{h_{\mathrm{s}}} R-q p k_{\mathrm{s}}^{2} h_{\mathrm{s}} & 0 \\
\frac{q p}{h_{\mathrm{s}}} R-q p k_{\mathrm{s}}^{2} h_{\mathrm{s}} & q^{2} k_{\mathrm{s}}^{2} h_{\mathrm{s}}+\frac{p^{2}}{h_{\mathrm{s}}} R & 0 \\
0 & 0 & \frac{s^{2}}{h_{\mathrm{p}}} R
\end{array}\right) .
$$

Here $R=4 s^{2} h_{\mathrm{p}} h_{\mathrm{s}}+\left(2 s^{2}-k_{\mathrm{s}}^{2}\right)^{2}$ is the Rayleigh function and $s^{2}=q^{2}+p^{2}$. The quadruple crack integrals are thus reduced to double Fourier integrals. These are slowly converging as they stand, but the following asymptotic behaviour of $S_{i m}$ for large $|q|$ with $p$ fixed (with obvious counterparts for large $|p|$ with $q$ fixed):

$$
16 \pi^{2} k_{\mathrm{s}}^{3} S_{i m}=C_{m} \delta_{i m}\left(|q|+\mathcal{O}\left(|q|^{-1}\right)\right)+\left(1-\delta_{i m}\right) \mathcal{O}(1),
$$

where $C_{1}=C_{3}=2 \mathrm{i}\left(k_{\mathrm{s}}^{2}-k_{\mathrm{p}}^{2}\right)$ and $C_{2}=\mathrm{i} k_{\mathrm{s}}^{2}$, may be exploited to improve the convergence. The asymptotes along the $q$ - and and $p$-axes are then subtracted and added back in the Fourier integrals, and the added back terms are evaluated analytically using the relations:

$$
\begin{aligned}
& \int_{-\infty}^{\infty} \frac{\mathrm{J}_{k}(q) \mathrm{J}_{l}(q)}{|q|} \mathrm{d} q=\frac{\delta_{k l}}{k}, \quad k, l=1,2, \ldots, \\
& \int_{-\infty}^{\infty} \frac{\mathrm{J}_{k}(q) \mathrm{J}_{l}(q)}{q^{2}} \mathrm{~d} q=-\frac{4\left(1+(-1)^{k+l}\right) \cos \left(\frac{\pi}{2}(k-l)\right)}{\pi\left(k^{4}+\left(l^{2}-1\right)^{2}-2 k^{2}\left(1+l^{2}\right)\right)}, \quad k, l=1,2, \ldots
\end{aligned}
$$

The remaining integrals, with the asymptotes subtracted, converge quickly enough for direct numerical integration using about 100 Gauss points in each direction in ordinary Gauss-Legendre quadrature.

In the computation of the crack signal response given by Eq. (4.3) the stress on the crack due to an incident field from the receiving probe, in the absence of the crack but with the back surface present, enters. This stress is computed by solving the back surface integral equation (3.1) with $\Delta u_{i}=0$ and then computing the stress from the integral representation, Eq. (3.5). Exactly the same integrals as in the crack BIE then enter, such that no additional integration is required in the computation of $\delta \Gamma_{C}$. The back surface signal response $\delta \Gamma_{B S}$, on the other hand, requires integration of the incident stress field (multiplied by the interpolation functions) on the boundary elements. Since this stress field is expressed as an inverse double Fourier transform, the computation of $\delta \Gamma_{B S}$ becomes very time consuming. In order to speed up these computations, the 2 D stationary phase approximation is used in the computation of the incident field given by Eq. (4.2). In the integral equations the incident displacement and stress fields also enter as load vectors, and the stationary phase approximation is used also for the computation of these. Applied to these cases the 2D stationary phase approximation takes the following form:

$$
\int_{C_{-}} f(\hat{\gamma}) \mathrm{e}^{\mathrm{i} k_{m} \boldsymbol{r} \cdot \hat{\boldsymbol{\gamma}}} \mathrm{d} \hat{\boldsymbol{\gamma}} \approx \frac{-2 \pi \mathrm{i}}{k_{m} r} f(\hat{\boldsymbol{r}}) \mathrm{e}^{\mathrm{i} k_{m} r} \text { as } k_{m} r \rightarrow \infty,
$$


where $r=|\boldsymbol{r}|, \hat{\boldsymbol{r}}=\boldsymbol{r} / r$ and $m=\mathrm{p}, \mathrm{s}$. For the validity of the stationary phase approximation several conditions should be met, as discussed in the paper by Boström [30] and Boström and Bövik [31]. In short, one may conclude that the crack and back surface should be at least a couple of wavelengths from the probe, and that the crack and probe diameters must not be too large compared to the distance from the probe to the crack. These conditions are usually satisfied in applications. The one most often violated is the condition on the probe size but in these cases the problem can be circumvented by dividing the probe surface into elements, see Ref. [31]. This probe element subdivision is employed also in the present model, and in the numerical examples presented in the next section a subdivision into 16 probe elements is used.

The discretization of the back surface yields a very large system matrix. This matrix may in general, as usual in the BEM, be both unsymmetric and fully populated. However, due to both the spatial decay due to geometrical spreading and the exponential decay due to material damping (which is included in the numerical examples) many off-diagonal matrix elements of the Green's tensors are very small. This can be exploited in a double thresholding scheme in such a way that all matrix elements that have a distance between the collocation point and element above a certain value are checked and set to zero if their value is below a threshold. The system matrix then becomes more or less sparse, and a sparse solver can be used, reducing the total computation times significantly. A similar method based on the same idea was previously employed by Orti, Alemán et al. [32], who note that for the elastic wave motion they consider the main features of the motion are preserved even for a threshold resulting in an approximated sparse system matrix containing less than $3 \%$ non-zero elements. For the considered examples in this paper a threshold resulting in a sparse system matrix with about $10 \%$ non-zero elements was used, and the equations were solved using the sparse parallel direct solver PARDISO [33].

In the computations to follow, a back surface size of approximately $15 \times$ 9 pressure wavelengths and boundary element lengths of $1 / 3$ of the Rayleigh wavelength were used. These values have been checked by changing both the back surface size and boundary element lengths to see that a proper convergence is obtained. For the back surface size it is noted that material damping is important in that it gives an exponential decay with distance, so the values chosen do depend on the damping. The boundary element length is rather large, but as long as the variations of the back surface are very smooth and the waves are predominantly shear waves (as the chosen probe is an SV probe) this gives good enough results. Unfortunately, there seem to be no other results, neither numerical nor experimental, that can be used to check the correctness of the results. However, time domain results (see the next section) are very good for checking the accuracy. If the back surface size is insufficient this will show up as artificial reflected pulses from the truncation boundaries of the back surface. An insufficient element size or other inaccuracies (computation of integrals, truncations in sums) generally give rise to noncausal responses. The time traces in the next section are casual, contain the expected pulses, and become very small once the dominant pulses have passed. 


\section{Numerical examples}

As stated in Section 2, the shape of the back surface may be quite arbitrary. For the numerical results presented in this section two simple examples of back surfaces are used as illustrations, the first being a smooth transition in the form of a quarter-wavelength sine function. The transition is from $x_{3}^{\mathrm{b}}=0$ to $x_{3}^{\mathrm{b}}=1.5 \mathrm{~mm}$ over the interval from $x_{1}^{\mathrm{b}}=1 \mathrm{~mm}$ to $x_{1}^{\mathrm{b}}=6 \mathrm{~mm}$, independently of $x_{2}^{\mathrm{b}}$. The second example is a planar back surface with a local, smooth bump in the form of a product of two half-wavelength sine functions. The height of the bump is $1.5 \mathrm{~mm}$ and it is located at $\left(x_{1}^{\mathrm{b}}, x_{2}^{\mathrm{b}}\right) \in[1,6] \times[-3,3] \mathrm{mm}$ so that the back surface is planar (with $x_{3}^{\mathrm{b}}=0$ ) outside this domain. The peak of the bump is thus located at $\boldsymbol{x}^{\mathrm{b}}=\{3.5,0,1.5\} \mathrm{mm}$. This second example is chosen to give an illustration of the influence on the signal response of a back surface with a very locally non-planar geometry. The crack is rectangular with sides $a_{1}=2 \mathrm{~mm}$ and $a_{2}=3 \mathrm{~mm}$ and the crack center located at $\boldsymbol{d}^{\mathrm{b}}=\{0,0,5\} \mathrm{mm}$. The Euler angles are $\varphi^{\mathrm{c}}=\psi^{\mathrm{c}}=0^{\circ}$ but $\theta^{\mathrm{c}}=90^{\circ}$ (the crack vertical) in Figs. 2 and 4 and $\theta^{\mathrm{c}}=45^{\circ}$ (the crack tilted $45^{\circ}$ ) in Figs. 3 and 5 .

For the steel material considered the Lamé constants are $\lambda=105 \mathrm{GPa}$, $\mu=76 \mathrm{GPa}$ and the density is $\rho=7900 \mathrm{~kg} / \mathrm{m}^{3}$, such that the wave propagation speeds are $c_{\mathrm{p}} \approx 5.7 \mathrm{~mm} / \mu \mathrm{s}$ and $c_{\mathrm{s}} \approx 3.1 \mathrm{~mm} / \mu \mathrm{s}$. To account for the effects of material damping the Lamé constants are given imaginary parts of $2 \%$ of the real parts.

In both cases the results of a pulse-echo testing situation are given, so the same probe is working as both transmitter and receiver. The probe is scanning along a surface parallel to the $x_{1}^{\mathrm{b}} x_{2}^{\mathrm{b}}$-plane and located at a distance of $20 \mathrm{~mm}$ from the lowermost part of the back surface. The probe is a square $10 \times 10 \mathrm{~mm}$ SV probe, angled $45^{\circ}$ to the right so that the probe angle $\gamma_{\mathrm{t}}=-45^{\circ}$ and with fluid coupling so $\delta=0$. The geometry of the probe, the crack and the non-planar part of the back surface is such that the crack is lying between the probe and the non-planar part, and thus 'shadows' the non-planar part, when the probe is located roughly at $-20<x_{1}^{\mathrm{b}}<-10 \mathrm{~mm}$.

In applications, calibration by a standard scatterer (typically a side-drilled hole) is usually performed. Here, such calibration is taken into account by computing the signal response from a side-drilled hole, and the maximum signal response obtained is then used as the calibration level when computing the pulseecho response as a function of probe position (but not in the time traces). In the calibration computation the transmitting and receiving probe models described in this paper are used. The scattering by the side-drilled hole is treated by the $T$ matrix method as discussed by Boström and Bövik [34], where more details are to be found. This method is essentially equivalent to separationof-variables, but the procedure also involves calculations of the field from the probe and transformation (translation and rotation) of this field to a cylindrical coordinate system in the side-drilled hole. Reciprocity is used to determine the response in the receiving probe, and the stationary phase approximation can be used to simplify the calculations and obtain quite explicit results.

Figures 2 and 3 show the pulse-echo signal response as a function of probe 
position for the two different crack orientations, for the case of the back surface with a transition from $x_{3}^{\mathrm{b}}=0$ to $x_{3}^{\mathrm{b}}=1.5 \mathrm{~mm}$. Figures $2(\mathrm{a})$ and $3(\mathrm{a})$ show the back surface signal responses, i.e. the responses in the absence of the crack, whereas Figs. 2(b) and 3(b) show the total signal responses from both the back surface and the crack. Figures 4 and 5 show the corresponding results for the second back surface example geometry, with a local bump. In all these figures the results are computed for a single frequency of $1 \mathrm{MHz}$, and the results are calibrated against a side-drilled hole of radius $2 \mathrm{~mm}$ located at a center depth of $20 \mathrm{~mm}$. A decibel (dB) scale is used in these figures, with a $40 \mathrm{~dB}$ difference between black (strongest, $8 \mathrm{~dB}$ over the calibration level) and white (weakest, $32 \mathrm{~dB}$ below the calibration level), in steps of $5 \mathrm{~dB}$.

As expected, the planar parts of the back surface give only a weak signal response, and this response is seen to be equal for the planar parts of the back surface in all the figures. The independence of $x_{2}^{\mathrm{b}}$ for the first back surface type is also clearly seen in the signal response in Figs. 2(a) and 3(a). For the considered crack orientations and back surface geometries there is also symmetry about the line $x_{2}^{\mathrm{b}}=0$, and this symmetry is apparent also in the signal response figures. Finally it can also be noted that the interaction of the back surface and the crack gives rise to quite complicated signal responses in Figs. 2(b), 3(b), 4(b) and 5(b). A 'shadowing' effect can be seen in Fig. 4(b) in that the response is weaker in the center of the plot where the crack lies in front of the non-planar back surface.

If the frequency spectra of the probes are known, the time traces can be computed by applying an inverse temporal Fourier transform. To model the spectrum of the probe in the numerical examples given here, a spectrum in the form of a Hanning window:

$$
\frac{1}{\Delta f} \cos ^{2}\left(\pi \frac{f-f_{c}}{2 \Delta f}\right)
$$

is assumed, where the center frequency $f_{c}=1 \mathrm{MHz}$ and the $6 \mathrm{~dB}$ bandwidth $\Delta f=0.5 \mathrm{MHz} .97$ frequencies are used in the numerical computation of the inverse temporal Fourier transform.

Figure 6 shows the time traces for a probe located at $\left(x_{1}^{\mathrm{b}}, x_{2}^{\mathrm{b}}\right)=(-30,0) \mathrm{mm}$, for both back surface geometries and both with and without the crack present, with crack angle $\theta^{c}=90^{\circ}$ (vertical crack). Figure 7 shows the corresponding result for a probe instead located at $\left(x_{1}^{\mathrm{b}}, x_{2}^{\mathrm{b}}\right)=(-15,0) \mathrm{mm}$. In both figures the same probe as above is used. The results in both these figures are normalized with the maximum in Figure $7(\mathrm{~d})$, but it should be noted that the scales are different in the two figures. The geometry in Fig. 6 is such that the main beam from the probe hits the back surface on the flat part in front of the non-planar part and the crack, but the reflection of the beam in the back surface hits the crack. In Fig. 7, on the other hand, the beam directly hits the crack and the non-planar part that is located directly behind the crack. The first contributions to the signal responses (which are relatively weak in Fig. 7) in these figures are the pulses from waves traveling vertically down to the back surface and back to the probe again, with arrival times of $t \approx 7.0 \mu \mathrm{s}, t \approx 10.0 \mu \mathrm{s}$ and $t \approx 12.9 \mu \mathrm{s}$ for 
the pure $\mathrm{P}$, mode converted and pure $\mathrm{S}$ wave pulses, respectively. It should be pointed out that this probe emits rather weak parts in the vertical direction, but due to the total reflection in the back surface, these pulses are still relatively strong. It may also seem that the first pulse arrives too early, around $t \approx 4.0 \mu \mathrm{s}$, but the reason for this early arrival is that the wave from the right side of the probe is emitted at time $t \approx-1.1 \mu$ s (so as to give an angled probe; time 0 is when the middle of the pulse is emitted from the middle of the probe). The same time difference is also valid upon reception of the pulse. Note also that due to the limited bandwidth, all pulses are 'smeared out' with widths of a few microseconds. In Fig. 6 also the reflections from the non-planar part of the back surface are visible, with corresponding arrival times of $t \approx 13.1 \mu \mathrm{s}, t \approx 18.6 \mu \mathrm{s}$ and $t \approx 24.1 \mu \mathrm{s}$. The main contribution from the crack is the corner echo (being reflected by both the crack and the back surface), with a wave path length corresponding to an arrival time of $t \approx 23.4 \mu$ s for the pure S-wave pulse which thus overlaps the last pulse from the non-planar part. With the crack present there are also possibilities of complex, multiple interactions between the crack and back surface resulting in pulses arriving late in the traces.

For the probe position $15 \mathrm{~mm}$ to the left of the crack center, Fig. 7, strong reflections from the non-planar parts of the back surfaces are expected. These reflections correspond to an arrival time of $t \approx 17.6 \mu$ s for the pure $\mathrm{S}$ wave pulse, and these pulses are the strongest ones seen in Fig. 7. The same vertically reflected back surface pulses as for the other probe position are of course present also in these traces, but for this probe position the different back surface geometries are also seen to influence the signal response differently; in Fig. 7(b) the total response is weaker than without the crack in Fig. 7(a), whereas the crack and back surface reflections interact to give an almost identical total response in Fig. 7(d) as compared to the results without the crack in Fig. 7(c). There seems to be no particular reason for this difference in behavior.

\section{Concluding remarks}

In this paper a complete model of ultrasonic nondestructive testing is developed. The model includes transmitting and receiving ultrasonic contact probes located on a thick-walled component with a non-planar back surface and an interior rectangular crack. The action of the transmitting probe is accounted for in a model based on prescribing the traction on the scanning surface, while an electromechanical reciprocity argument is used to model the receiving probe and yield expressions for the signal response due to the crack and back surface. The wave scattering problem is solved by reformulating it as two coupled boundary integral equations for the unknown crack opening and back surface displacements. By using a combination of a series expansion of the crack opening displacement and a boundary element discretization of the back surface to solve the coupled integral equations, the hypersingularity in the crack BIE can be treated analytically while the back surface is allowed to be of an arbitrary, complex geometry. The discretization of the back surface generates many unknowns 
and leads to demanding computations, but the use of a fast sparse solver is enabled by applying a threshold criterion to generate a sparse approximation of the system matrix. The computations are accelerated further by employing the stationary phase approximation for the computation of all probe field integrals. Material damping and calibration against a side-drilled hole is included in the model, and time traces are computed by applying an inverse temporal Fourier transform. A few numerical results are given, illustrating the possibilities of the model and the effects of the back surface geometry on the wave scattering.

\section{Acknowledgments}

A grateful acknowledgement is directed towards Prof. Andrés Saéz, Universidad de Sevilla, Spain, for providing the one of the authors (J. W.) with an opportunity to stay in Seville for one month and work in an environment of active BEM-researchers.

The computations for the numerical examples presented in this paper were performed on resources provided by the Swedish National Infrastructure for Computing (SNIC) at C3SE.

The work presented in this paper is sponsored by the Swedish Radiation Safety Authority (SSM) and this is gratefully acknowledged.

\section{References}

[1] R. Thompson, T. Gray, Use of ultrasonic models in the design and validation of new NDE techniques, Proc. R. Soc. London A 320 (1986) 329-340.

[2] J. Achenbach, Measurement models for quantitative ultrasonics, J. Sound Vib. 159 (1992) 385-401.

[3] J. Achenbach, Quantitative nondestructive evaluation, Int. J. Solids Struct. 37 (2000) 13-27.

[4] J. Achenbach, Modeling for quantitative non-destructive evaluation, Ultrasonics 40 (2002) 1-10.

[5] P. Calmon, A. Lhémery, I. Lecæur-Taïbi, R. Raillon, L. Paradis, Models for the computation of ultrasonic fields and their interaction with defects in realistic NDT configurations, Nucl. Eng. Des. 180 (1998) 271-283.

[6] R. Chapman, A system model for the ultrasonic inspection of smooth planar cracks, J. Nondestr. Eval. 9 (1990) 197-210.

[7] P. Schafbuch, F. Rizzo, R. Thompson, Application of the boundary element method to elastic wave scattering by irregular defects, J. Nondestr. Eval. 9 (1990) 113-127.

[8] S. Ström, Introduction to integral representations and integral equations for time-harmonic acoustic, electromagnetic and elastodynamic wave fields, in: Varadan et al. [35]. 
[9] M. Bonnet, Boundary Integral Equation Methods for Solids and Fluids, John Wiley \& Sons Ltd, Chichester, 1995.

[10] M. Guiggiani, Formulation and numerical treatment of boundary integral equations with hypersingular kernels, in: Sladek and Sladek [36].

[11] J. Westlund, A. Boström, A 2D model of ultrasonic testing for cracks near a non-planar surface, Wave Motion 47 (2010) 1103-1112.

[12] Y.-H. Pao, V. Varatharajulu, Huygen's principle, radiation conditions, and integral formulas for the scattering of elastic waves, J. Acoust. Soc. Am. 59 (1976) 1361-1371.

[13] C. Zhang, D. Gross, On Wave Propagation in Elastic Solids with Cracks, Computational Mechanics Publications, Southampton, 1998.

[14] V. Sladek, J. Sladek, Introductory notes on singular integrals, in: Singular Integrals in Boundary Element Methods [36].

[15] P. Martin, F. Rizzo, On boundary integral equations for crack problems, Proc. R. Soc. London A 421 (1989) 341-355.

[16] P. Martin, F. Rizzo, Hypersingular integrals: How smooth must the density be?, Int. J. Numer. Methods Eng. 39 (1996) 687-704.

[17] A. Boström, H. Wirdelius, Ultrasonic probe modeling and nondestructive crack detection, J. Acoust. Soc. Am. 97 (1995) 2836-2848.

[18] A. Boström, G. Kristensson, S. Ström, Transformation properties of plane, spherical and cylindrical scalar and vector wave functions, in: Varadan et al. [35].

[19] B. Auld, General electromechanical reciprocity relations applied to the calculation of elastic wave scattering coefficients, Wave Motion 1 (1979) 3-10.

[20] R. Thompson, Interpretation of Auld's electromechanical reciprocity relation via a one-dimensional example, Res. Nondestr. Eval. 5 (1994) 147-155.

[21] J. Mattsson, A. J. Niklasson, Ultrasonic 2-D SH crack detection in anisotropic solids, J. Nondestr. Eval. 16 (1997) 31-41.

[22] A. Boström, Acoustic scattering by a sound-hard rectangle, J. Acoust. Soc. Am. 99 (1991) 3344-3347.

[23] L. Guan, A. Norris, Elastic wave scattering by rectangular cracks, Int. J. Solids Struct. 29 (1992) 1549-1565.

[24] J. Helsing, A. Jonsson, G. Peters, Evaluation of the mode I stress intensity factor for a square crack in 3D, Eng. Fract. Mech. 68 (2001) 605-612.

[25] N. Nishimura, S. Kobayashi, A regularized boundary integral equation method for elastodynamic crack problems, Comp. Mech. 4 (1989) 319-328. 
[26] J. Domínguez, Boundary Elements in Dynamics, Computational Mechanics Publications, Southampton, 1993.

[27] G. Beer, Programming the Boundary Element Method, John Wiley \& Sons Ltd, Chichester, 2001.

[28] M. Rezayat, D. Shippy, F. Rizzo, On time-harmonic elastic-wave analysis by the boundary element method for moderate to high frequencies, Comp. Meth. Appl. Mech. Engng. 55 (1986) 349-367.

[29] J. Westlund, 2D SH modelling of ultrasonic testing for cracks near a nonplanar surface, Eng. Anal. Boundary Elem. 33 (2009) 1103-1112.

[30] A. Boström, Review of hypersingular integral equation method for crack scattering and application to modeling of ultrasonic non-destructive evaluation, Appl. Mech. Rev. 56 (2003) 383-405.

[31] A. Boström, P. Bövik, Ultrasonic scattering by a side-drilled hole, Int. J. Solids Struct. 40 (2003) 3493-3505.

[32] C. Ortiz-Alemán, F. Sánches-Sesma, J. Rodríguez-Zúñiga, F. Luzón, Computing topographical 3D site effects using a fast IBEM/conjugate gradient approach, Bull. Seis. Soc. Am. 88 (1998) 393-399.

[33] O. Schenk, K. Gärtner, Solving unsymmetric sparse systems of linear equations with PARDISO, J. Fut. Gen. Comp. Sys. 20 (2004) 475-487.

[34] A. Boström, P. Bövik, Ultrasonic scattering by a side-drilled hole, Int. J. Solids Struct. 40 (2003) 3493-3505.

[35] V. Varadan, A. Lakhtakia, V. Varadan (Eds.), Field Representations and Introduction to Scattering, Vol. 1 of Acoustic, electromagnetic and elastic wave scattering, North-Holland, Amsterdam, 1991.

[36] V. Sladek, J. Sladek (Eds.), Singular Integrals in Boundary Element Methods, Advances in Boundary Element Series, Computational Mechanics Publications, Southampton, 1998. 


\section{List of Figures}

1 The geometry with an interior rectangular crack in a thick-walled component with a non-planar back surface. . . . . . . . . . 21

2 The echo amplitude as a function of probe position, back surface with transition. Crack angle $\theta^{\mathrm{c}}=90^{\circ}$. . . . . . . . . . 22

3 The echo amplitude as a function of probe position, back surface with transition. Crack angle $\theta^{\mathrm{c}}=45^{\circ}$. . . . . . . . . . 23

4 The echo amplitude as a function of probe position, back surface with local bump. Crack angle $\theta^{\mathrm{c}}=90^{\circ}$. . . . . . . . . . . . 24

5 The echo amplitude as a function of probe position, back surface with local bump. Crack angle $\theta^{\mathrm{c}}=45^{\circ}$. . . . . . . . 25

6 Time traces for probe position $\left(x_{1}^{\mathrm{b}}, x_{2}^{\mathrm{b}}\right)=(-30,0) \mathrm{mm}$, for the two different example back surface geometries. Crack angle $\theta^{\mathrm{c}}=$

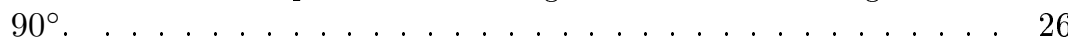

$7 \quad$ Time traces for probe position $\left(x_{1}^{\mathrm{b}}, x_{2}^{\mathrm{b}}\right)=(-15,0) \mathrm{mm}$, for the two different example back surface geometries. Crack angle $\theta^{\mathrm{c}}=$

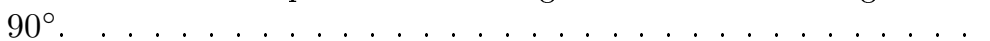




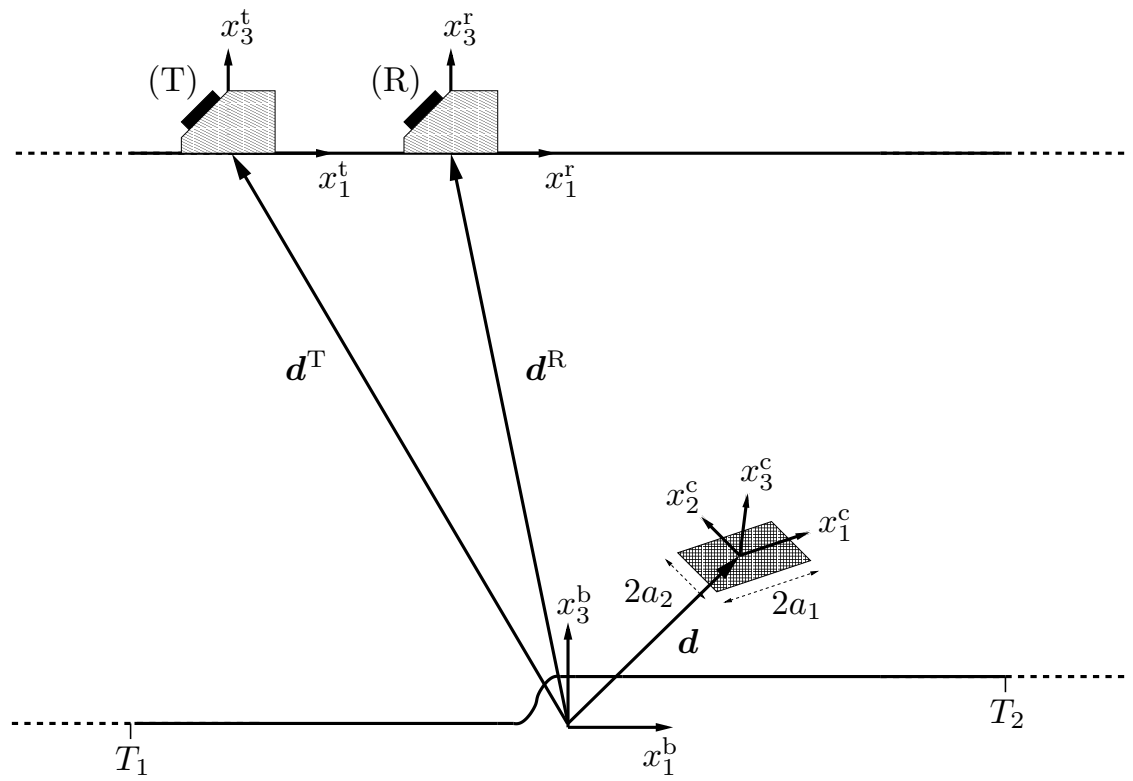

Figure 1: The geometry with an interior rectangular crack in a thick-walled component with a non-planar back surface. 


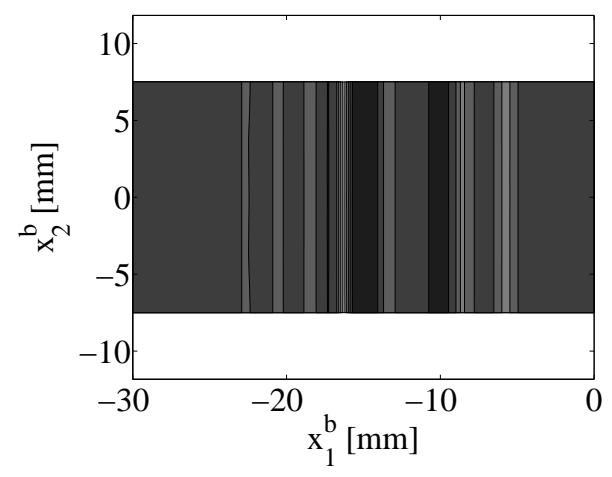

(a) Back surface signal response.

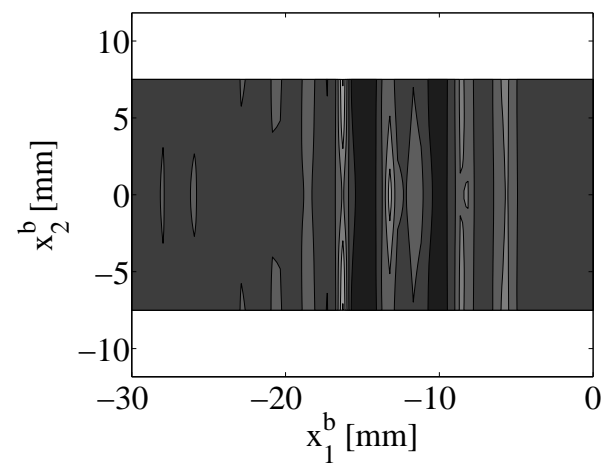

(b) Total signal response.

Figure 2: The echo amplitude as a function of probe position, back surface with transition. Crack angle $\theta^{\mathrm{C}}=90^{\circ}$. 


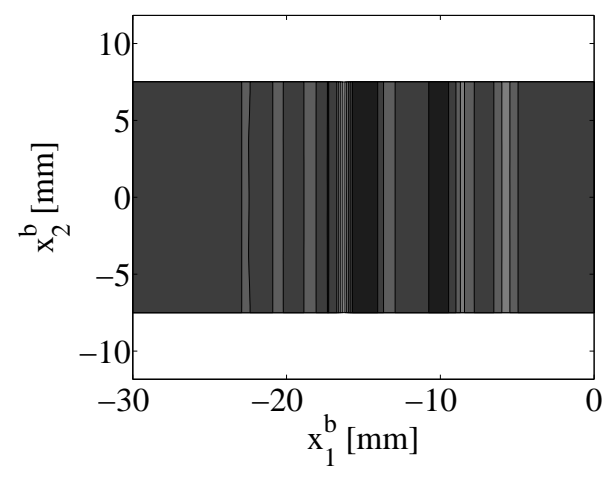

(a) Back surface signal response.

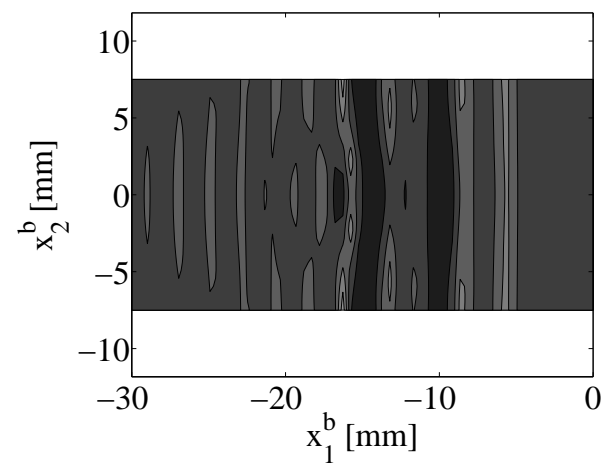

(b) Total signal response.

Figure 3: The echo amplitude as a function of probe position, back surface with transition. Crack angle $\theta^{\mathrm{C}}=45^{\circ}$. 


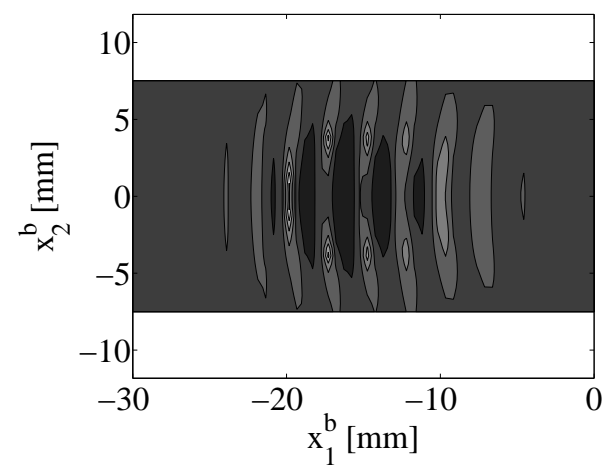

(a) Back surface signal response.

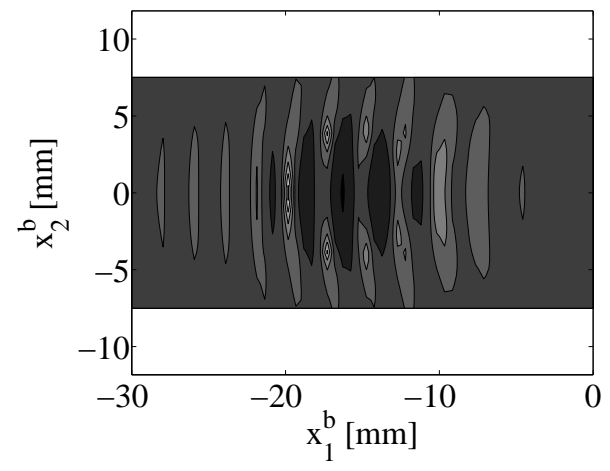

(b) Total signal response.

Figure 4: The echo amplitude as a function of probe position, back surface with local bump. Crack angle $\theta^{\mathrm{C}}=90^{\circ}$. 


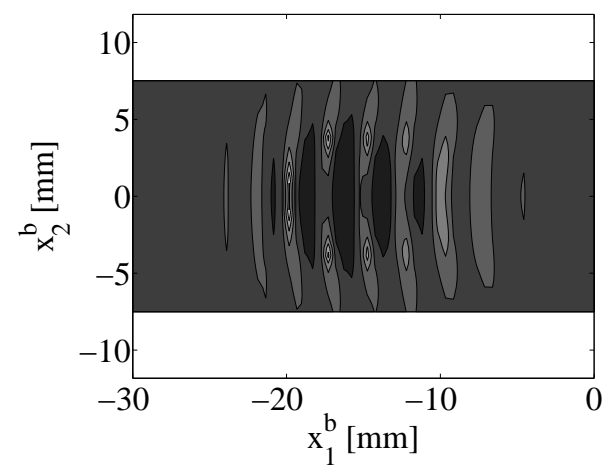

(a) Back surface signal response.

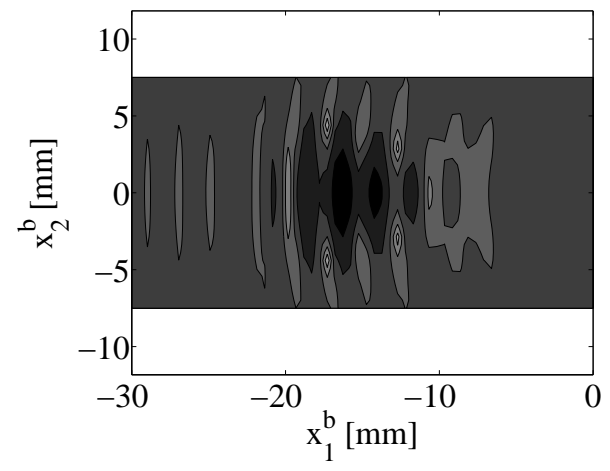

(b) Total signal response.

Figure 5: The echo amplitude as a function of probe position, back surface with local bump. Crack angle $\theta^{\mathrm{c}}=45^{\circ}$. 


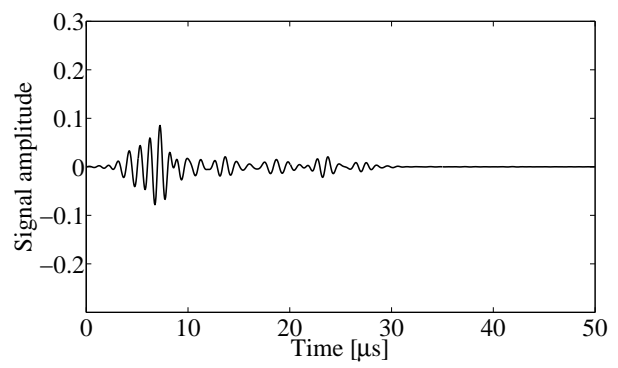

(a) Back surface with transition, crack absent.

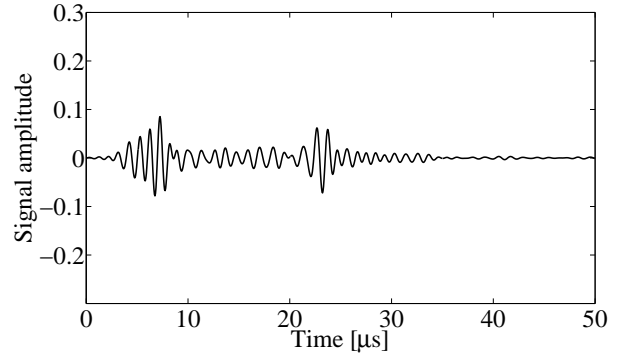

(b) Back surface with transition, crack present.

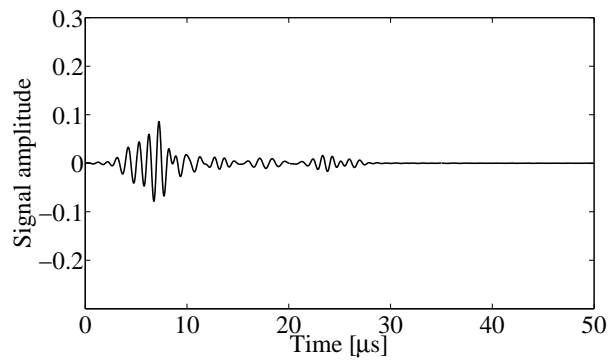

(c) Back surface with local bump, crack absent.

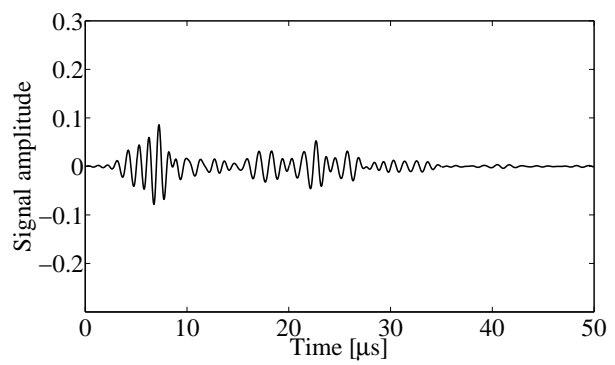

(d) Back surface with local bump, crack present.

Figure 6: Time traces for probe position $\left(x_{1}^{\mathrm{b}}, x_{2}^{\mathrm{b}}\right)=(-30,0) \mathrm{mm}$, for the two different example back surface geometries. Crack angle $\theta^{\mathrm{c}}=90^{\circ}$. 


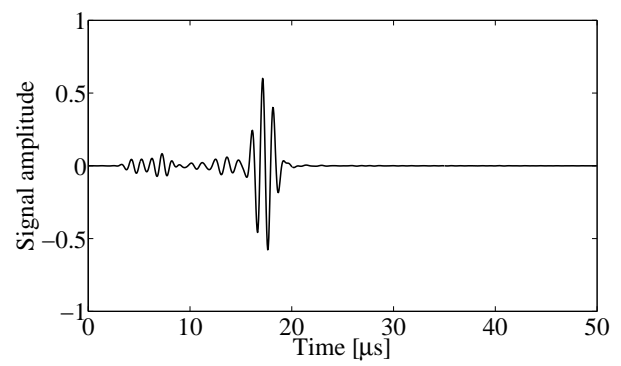

(a) Back surface with transition, crack absent.

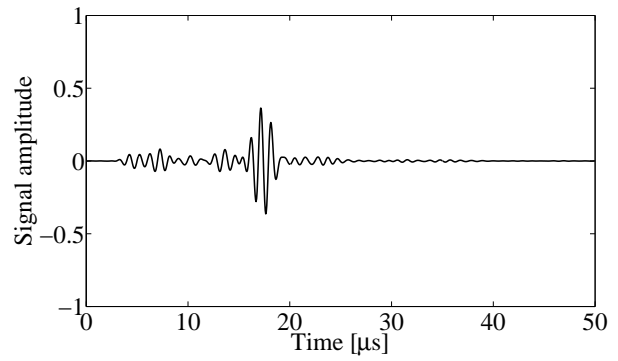

(b) Back surface with transition, crack present.

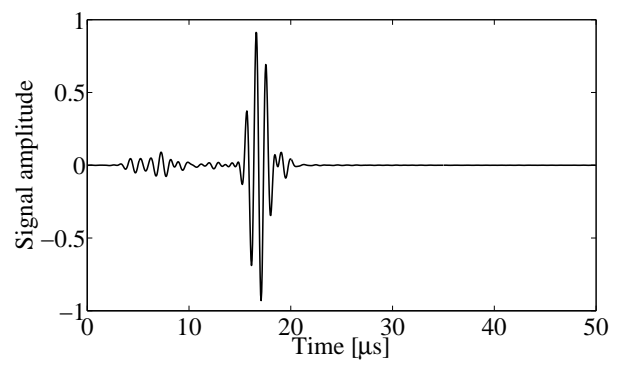

(c) Back surface with local bump, crack absent.

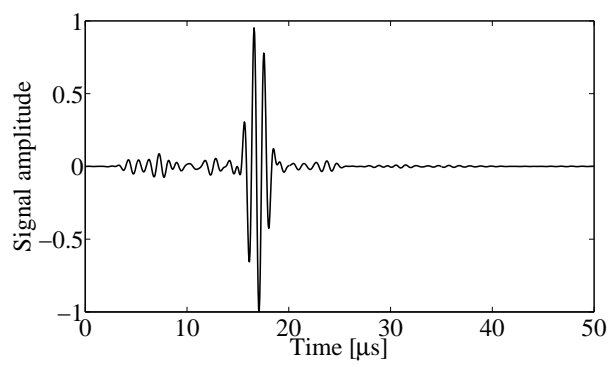

(d) Back surface with local bump, crack present.

Figure 7: Time traces for probe position $\left(x_{1}^{\mathrm{b}}, x_{2}^{\mathrm{b}}\right)=(-15,0) \mathrm{mm}$, for the two different example back surface geometries. Crack angle $\theta^{\mathrm{c}}=90^{\circ}$. 